\title{
Multi-wavelength study of the G $82.2+5.3$ supernova remnant
}

\author{
F. Mavromatakis ${ }^{1}$, B. Aschenbach ${ }^{2}$, P. Boumis ${ }^{1, \star}$, and J. Papamastorakis ${ }^{1,3}$ \\ 1 University of Crete, Physics Department, PO Box 2208, 71003 Heraklion, Crete, Greece \\ ${ }^{2}$ Max-Planck-Institut für extraterrestrische Physik, Postfach 1312, 85741 Garching, Germany \\ ${ }^{3}$ Foundation for Research and Technology-Hellas, PO Box 1527, 71110 Heraklion, Crete, Greece
}

Received 8 August 2003 / Accepted 6 November 2003

\begin{abstract}
We present the first CCD flux-calibrated images of the supernova remnant $\mathrm{G} 82.2+5.3$ in major optical emission lines. The medium ionization line of [O III]5007 $\AA$ provides the first direct evidence of optical emission originating from G 82.2+5.3. Filamentary emission is detected in the west and east areas of the remnant, roughly defining an ellipsoidal shell. The [O III] emission is rather well correlated with the radio emission suggesting their association, while typical fluxes are found in the range of 20-30 $\times 10^{-17} \mathrm{erg} \mathrm{s}^{-1} \mathrm{~cm}^{-2} \operatorname{arcsec}^{-2}$. Deep long-slit spectra taken at specific positions of the remnant verify that the detected filamentary emission originates from shock heated gas, while the diffuse [O III] emission in the south results from photoionization processes. The spectra further suggest shock velocities around $100 \mathrm{~km} \mathrm{~s}^{-1}$ and low electron densities. The X-ray surface brightness is quite patchy, missing obvious limb brightening and is dominated by a bright bar-like emission region which is off-set from the geometric center by $\sim 9^{\prime}$. The X-ray emission is thermal and requires two temperatures of $0.2 \mathrm{keV}$ and $0.63 \mathrm{keV}$. The bright bar region shows overabundant $\mathrm{Mg}, \mathrm{Si}$ and $\mathrm{Fe}$, which might indicate still radiating ejecta matter. The azimuthally averaged radial surface profile is consistent with the matter density changing with distance $r$ from the center $\propto \mathrm{e}^{-r / r_{0}}$ with a characteristic angular length of $36^{\prime}$, or, alternatively, with an $r^{-1 / 2}$ density profile. The matter inside the remnant is quite likely structured like a porous cloudy medium. The average matter density is $\sim 0.04 \times d_{1.6}^{-0.5}$ with $d_{1.6}$ the distance in units of $1.6 \mathrm{kpc}$. Because of the low density and the long cooling times involved the remnant is more likely to be in the adiabatic phase, which is consistent with the densities derived for the X-ray plasma and the optical line emission, but it is not excluded that is has reached the radiating phase. This, however, would imply a lower density, greater age and much larger distance, at the edge of the upper limits obtained from $N_{\mathrm{H}}$ and $E(B-V)$.
\end{abstract}

Key words. ISM: general - ISM: supernova remnants - ISM: individual objects: G 82.2+5.3

\section{Introduction}

The supernova remnant G 82.2+5.3 (W 63) is found in the complex region of the Cygnus constellation. Radio observations at several frequency bands established the nature of the object through its non-thermal radio spectrum (Velusamy \& Kundu 1974; Angerhofer et al. 1977; Wendker 1971). The remnant displays a non-circular, elliptical shape of $\sim 70^{\prime} \times 100^{\prime}$ in the radio map at $11 \mathrm{~cm}$. The distance to the remnant is not well determined and distances in the range of 1.3-1.9 kpc have been reported (Rosado \& Gonzalez 1981, and references therein). Interference-filter photographs from Parker et al. (1979) revealed bright extended structures in $\mathrm{H} \alpha$ and [S II]. There is no obvious correlation between the low ionization images and the radio maps of $\mathrm{G} 82.2+5.3$. Optical spectra taken from Sabbadin (1976) show the characteristic signature of emission from shock heated gas ([S II $] / \mathrm{H} \alpha \sim 0.5)$. Rosado \& Gonzalez (1981) estimated expansion velocities for some of the optical

Send offprint requests to: F. Mavromatakis, e-mail: fotis@physics.uoc.gr

* Present address: Institute of Astronomy \& Astrophysics, National Observatory of Athens, I. Metaxa \& V. Pavlou, P. Penteli, 15236 Athens, Greece. filaments of $\sim 35 \mathrm{~km} \mathrm{~s}^{-1}$ and $\sim 70 \mathrm{~km} \mathrm{~s}^{-1}$, depending upon the size assumed for the remnant.

The X-ray and radio morphologies of the remnant are quite different. Rho \& Petre (1998) proposed that it belongs to the class of mixed-morphology remnants. Indeed, the X-ray emission is neither shell-like nor plerion-like but the vast majority of the X-rays originate from areas around but not peaked on the remnant center, and there is no obvious limb brightening. The entire X-ray region is roughly bound by the radio shell. Rho \& Petre (1998) analysing ROSAT data describe the entire spectrum by a thermal, single temperature spectrum with $k T \sim 0.2 \mathrm{keV}$ subjected to interstellar absorption with a column density of $\sim 4 \times 10^{21} \mathrm{~cm}^{-2}$ although with large uncertainties.

Flux calibrated images in major optical emission lines were obtained and deep long-slit spectroscopy of selected areas of interest was performed for a detailed study of the optical emission in the area of $\mathrm{G} 82.2+5.3$. In addition, we analyzed ROSAT All-Sky survey and re-analyzed ROSAT pointed data as well as ASCA GIS and SIS data from one pointing. Information about the observations and the data reduction is given in Sect. 2. In Sects. 3 and 4 we present the results of our imaging and spectral observations. Finally, in Sect. 5 we discuss the properties of the remnant and its environment. 
Table 1. Journal of the observations.

\begin{tabular}{lllll}
\hline \hline Filter & $\begin{array}{l}\lambda_{\mathrm{C}} \\
(\AA)\end{array}$ & $\begin{array}{l}\Delta \lambda \\
(\AA)\end{array}$ & Date (UT) & $\begin{array}{l}\text { Exp. times }^{a} \\
(\text { No of frames }\end{array}$ \\
\hline $\mathrm{H} \alpha+[\mathrm{N} \mathrm{II}]$ & 6560 & 75 & $25-26 / 07 / 2001$ & $7200(3)$ \\
{$[\mathrm{S}$ II $]$} & 6708 & 20 & $25 / 07 / 2001$ & $7200(3)$ \\
{$[\mathrm{O}$ III $]$} & 5005 & 28 & $26 / 07 / 2001$ & $9600(4)$ \\
{$[$ O II $]$} & 3727 & 25 & $26 / 07 / 2001$ & $7200(3)$ \\
Cont red & 6096 & 134 & $25-26 / 07 / 2001$ & $720(8)$ \\
Cont blue & 5470 & 230 & $26 / 07 / 2001$ & $360(2)$ \\
\hline
\end{tabular}

${ }^{a}$ Total exposure times in $\mathrm{s}$.

${ }^{b}$ Number of individual frames.

\section{Observations}

\subsection{Optical images}

The current wide field observations were performed with the $0.3 \mathrm{~m}$ Schmidt-Cassegrain telescope at Skinakas Observatory, Crete, Greece. The complex field of G 82.2+5.3 was observed on July 25 and 26,2001 . The $1024 \times 1024$ SITe CCD used during these imaging observations resulted in a $89^{\prime} \times 89^{\prime}$ field of view and an image scale of $5^{\prime \prime}$ per pixel. A journal of the observations together with information about the filters are given in Table 1. The images presented in this work are the average of the available frames from the individual filters. The astrometric solutions for all data frames utilized the HST Guide star catalogue (Lasker et al. 1999) and all coordinates quoted in this work refer to epoch 2000. All frames were projected to a common origin on the sky.

Standard IRAF and MIDAS routines were employed for the reduction of the data. All frames were bias subtracted and flat-field corrected using a series of well exposed twilight flat-fields. The spectrophotometric standard stars HR 5501, HR 7596, HR 7950 and HR 9087 were observed in order to obtain absolute flux calibration (Hamuy et al. 1992, 1994).

\subsection{Optical spectra}

The $1.3 \mathrm{~m}$ Ritchey-Cretien telescope at Skinakas Observatory was used to obtain long-slit spectra on June 21, 22, and July 21, 22 2001. The data were taken with a 1300 line $\mathrm{mm}^{-1}$ grating and a $800 \times 2000$ SITe CCD covering the range of 4750 $6815 \AA$. The slit had a width of 7'.7 and a length of 7!9 and, in all cases, was oriented in the south-north direction. The coordinates of the slit centers, the number of available spectra from each location and the total exposure time of each spectrum are given in Table 2. The spectrophotometric standard stars HR 5501, HR 7596, HR 9087, HR 718 and HR 7950 were observed for the absolute calibration of the spectra of G 82.2+5.3.

\subsection{The ROSAT and ASCA observations}

The Cygnus region was scanned during the ROSAT All-sky survey in early December 1992. X-rays from G 82.2+5.3, which is located at the northern tip of the so-called Cygnus Superbubble shell (Uyaniker et al. 2001) were clearly detected and an image of the remnant and the environment
Table 2. Spectral log.

\begin{tabular}{lll}
\hline \hline Slit centers & & Exp. times $^{a}$ \\
\hline$\alpha$ & $\delta$ & No of spectra $^{b}$ ) \\
\hline $20^{\mathrm{h}} 21^{\mathrm{m}} 39^{\mathrm{s}} .3$ & $45^{\circ} 59^{\prime} 30^{\prime} \cdot 1$ & $16500(5)$ \\
$20^{\mathrm{h}} 20^{\mathrm{m}} 04^{\mathrm{s}} .0$ & $44^{\circ} 54^{\prime} 48^{\prime \prime} .5$ & $7200(2)$ \\
$20^{\mathrm{h}} 16^{\mathrm{m}} 15^{\mathrm{s}} .6$ & $45^{\circ} 51^{\prime} 58^{\prime \prime} \cdot 9$ & $7200(2)$ \\
$20^{\mathrm{h}} 16^{\mathrm{m}} 15^{\mathrm{s}} .4$ & $45^{\circ} 58^{\prime} 01^{\prime \prime} 4$ & $7200(2)$ \\
\hline & \\
${ }^{a}$ Number of spectra obtained. & \\
${ }^{b}$ Total exposure times in sec. &
\end{tabular}

could be constructed. The net observing time was $\sim 1200 \mathrm{~s}$. The remnant under study was also observed several times with the ROSAT PSPC in pointing mode, the longest observation of which was for $\sim 8 \mathrm{ks}$ on 26 October 1993 aiming at the center of the remnant (sequence \#500286p). Additional ROSAT observations were targeted at the west of the remnant (\#500219p/500219p1), the north-east (\#500217p) and the south-east (\#500218p). Apart from the pointing towards the centre only the south-east pointing provided sufficient counts to create a spectrum over the central 40 arcmin of the PSPC. We also found one observation in the ASCA archive and both the GIS and SIS data could be used for a spectral analysis.

\section{The optical emission}

\subsection{The $H \alpha+[N 川]$ and [S II] line images}

The images in $\mathrm{H} \alpha+[\mathrm{NII}]$ (Fig. 1) and [S II] (Fig. 2) of the remnant look complex due to the presence of several bright and dark nebulae. The dark nebulae LDN 908 and 910 (Lynds 1962) are found in the south areas of our field, while LBN 081.88+04.79 (LBN 314; Lynds 1965) is located in the south-east and LBN 082.79+05.83 (LBN 325), LBN 083.03+05.78 (LBN 326) located to the north may contribute to the emission in our field. Lynds (1965) also reports LBN 081.62+05.47 (LBN 310) as a very extended H II region which seems to overlap our field. The complexity of the environment of the remnant does not allow the direct identification of any optical emission matching the morphology of the known radio emission. However, since our images are flux calibrated they can be used to approximately determine the emission characteristics. The ratio of the low ionization images of [S II] and $\mathrm{H} \alpha+[\mathrm{N} \mathrm{II}]$ shows that the bulk of the emission in the west results from photoionization, while the weak emission seen to the south of the bright star GSC 3572-2102 probably originates from shock heated gas. In this area, roughly defined along $\alpha \simeq$ $20^{\mathrm{h}} 22^{\mathrm{m}} 30^{\mathrm{s}}$ and between $\delta \simeq 45^{\circ} 47^{\prime}$ and $45^{\circ} 12^{\prime}$, we estimate a $[\mathrm{S}$ II] $/ \mathrm{H} \alpha$ ratio of $\sim 0.7-0.8$, while $\mathrm{LBN} 082.79+05.83$ and the bright structure in the north-west are characterized by ratios very close to $\sim 0.4$.

\subsection{The $[O ॥]$ and $[\mathrm{O} I I]$ images}

The [O II] image of the area around G 82.2+5.3 is not shown here since its morphology is generally similar to that of the $\mathrm{H} \alpha+[\mathrm{N} \mathrm{II}]$ and $[\mathrm{S} \mathrm{II}]$ low ionization images. Emission from the 


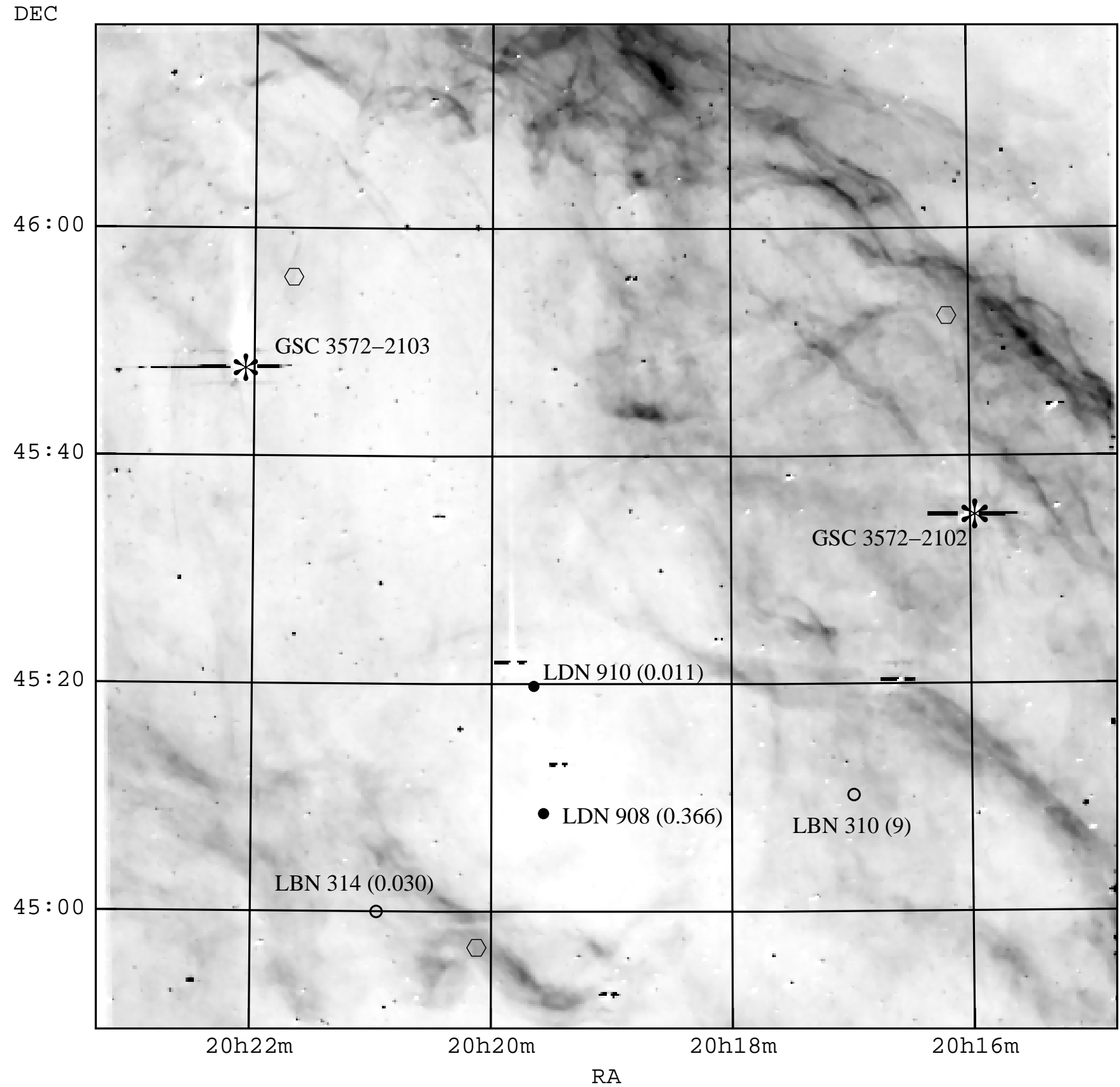

Fig. 1. The field of G 82.2+5.3 in the $\mathrm{H} \alpha+[\mathrm{N}$ II] filter. The image has been smoothed to suppress the residuals from the imperfect continuum subtraction. The open and the filled circles designate the positions of bright and dark nebulae, respectively, while the numbers in parentheses represent the surface of each nebula in square degrees as given by Lynds $(1962,1965)$. The open polygons show the positions where emission line ratios are measured (see also Sect. 3.3). The shadings run linearly from 0 to $400 \times 10^{-17} \mathrm{erg} \mathrm{s}^{-1} \mathrm{~cm}^{-2} \operatorname{arcsec}^{-2}$. The line segments seen near over-exposed stars in this figure and the next figures are due to the blooming effect.

H II region in the [O II $] 3727 \AA$ A line is somewhat suppressed but still the shape of the remnant is not clearly outlined. No immediate correlation of the optical emission with the known radio emission can be seen. In strong contrast to the low ionization images, the field of G 82.2+5.3 appears markedly different in the medium ionization line of [O III] $5007 \AA$ (Fig. 3). The overall emission is significantly attenuated and the image appears to be free of complex structures. Optical radiation possibly from LBN $082.79+05.83$ and/or $083.03+05.78$ is seen in the north edge of the field of view. The [O III] image reveals filamentary emission in the west and east areas as well as diffuse emission. Bright filaments are detected along $\alpha \simeq 20^{\mathrm{h}} 16^{\mathrm{m}}$ and $20^{\mathrm{h}} 22^{\mathrm{m}}$ and around $\delta \simeq 45^{\circ} 40^{\prime}$. These two filamentary structures define rather well the opposite sides of an ellipsoidal shell which is in agreement with the morphology of the radio emission. In fact the correlation of the [O III] emission in the east with the radio emission observed at $326 \mathrm{MHz}$ and $4850 \mathrm{MHz}$ (Fig. 4) suggests its association to $\mathrm{G} 82.2+5.3$.

Fainter structures are also detected in the south and inner regions of the field. Faint emission from a region shaped as an 


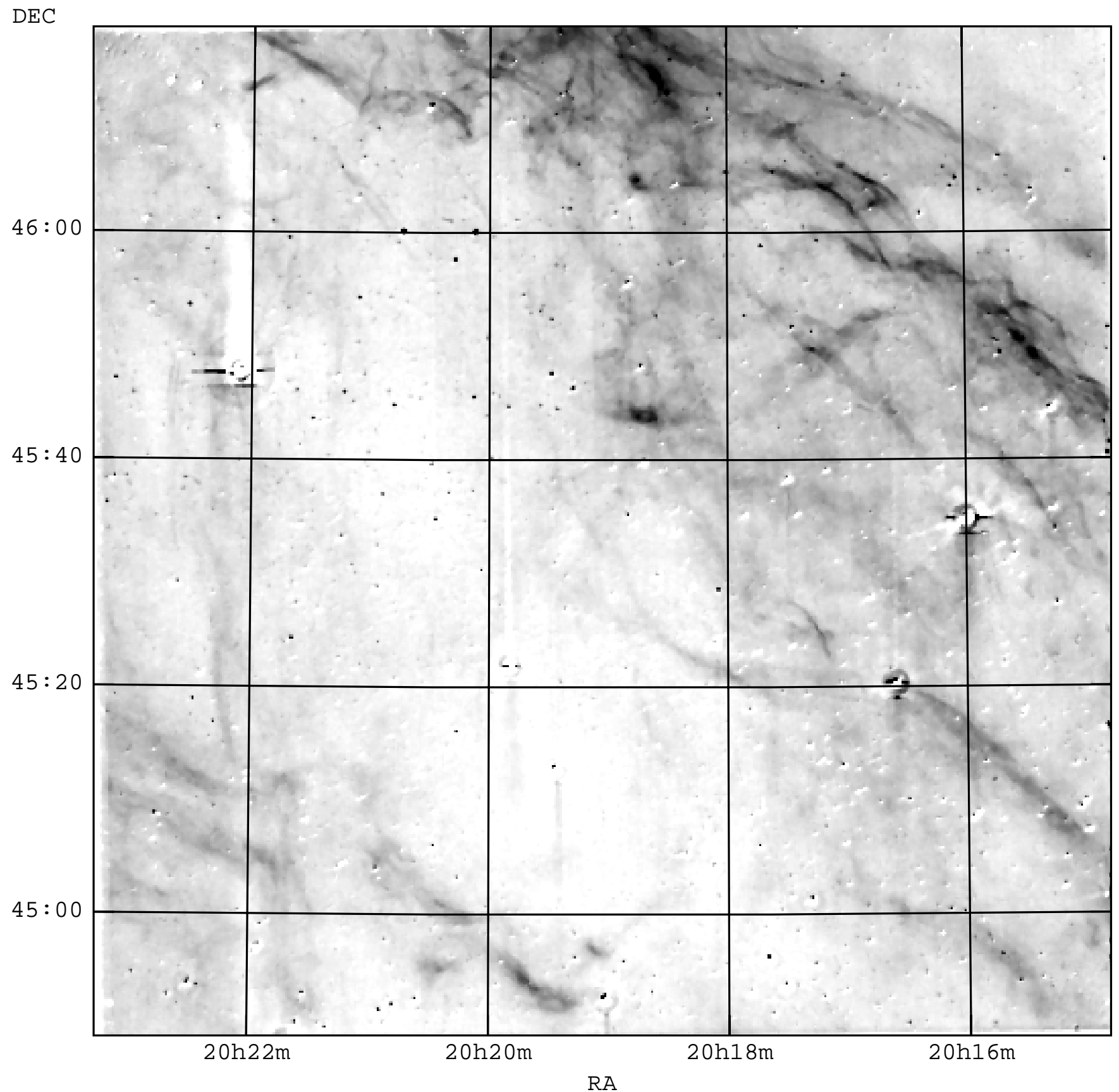

Fig. 2. The [S II] image of the area around G 82.2+5.3. The morphology in these emission lines is similar to that of the H $\alpha+[\mathrm{N}$ II] lines. The image has been smoothed to suppress the residuals from the imperfect continuum subtraction, while the shadings run linearly from 0 to $60 \times$ $10^{-17} \mathrm{erg} \mathrm{s}^{-1} \mathrm{~cm}^{-2} \operatorname{arcsec}^{-2}$.

arc is detected to the east of GSC 3572-2102, roughly along $\alpha \simeq 20^{\mathrm{h}} 22^{\mathrm{m}} 40^{\mathrm{s}}$, from $\delta \simeq 45^{\circ} 30^{\prime}$ to $46^{\circ} 30^{\prime}$. This faint filamentary structure is not continuous but gaps in intensity are detected along its extent. This may indicate the presence of inhomogeneities in the interstellar "clouds" resulting in strong variations of the shock velocity. It is known that the shock velocity greatly influences the [O III] flux (Cox \& Raymond 1985). The typical projected angular width of the [O III] filaments is $\sim 30^{\prime \prime}$, equivalent to $0.2 \mathrm{pc}$ at an assumed average distance of $1.6 \mathrm{kpc}$ (see Sect. 1).

\subsection{The long-slit spectra from $G 82.2+5.3$}

The deep low resolution spectra were taken in the vicinity of the bright stars GSC 3572-2102 and GSC 3572-2103, as well as in the south in an area where strong radio emission overlaps with strong $\mathrm{H} \alpha+[\mathrm{N} \mathrm{II}]$ and diffuse [O III] emission (Fig. 1). The optical spectra from areas I and II (Fig. 3) indicate emission from shock heated gas ( $[\mathrm{S} \mathrm{II}] / \mathrm{H} \alpha>0.4)$, while that from area III seems to originate from photoionized gas (Table 3). This is because we cannot exclude the possibility that the spectrum 

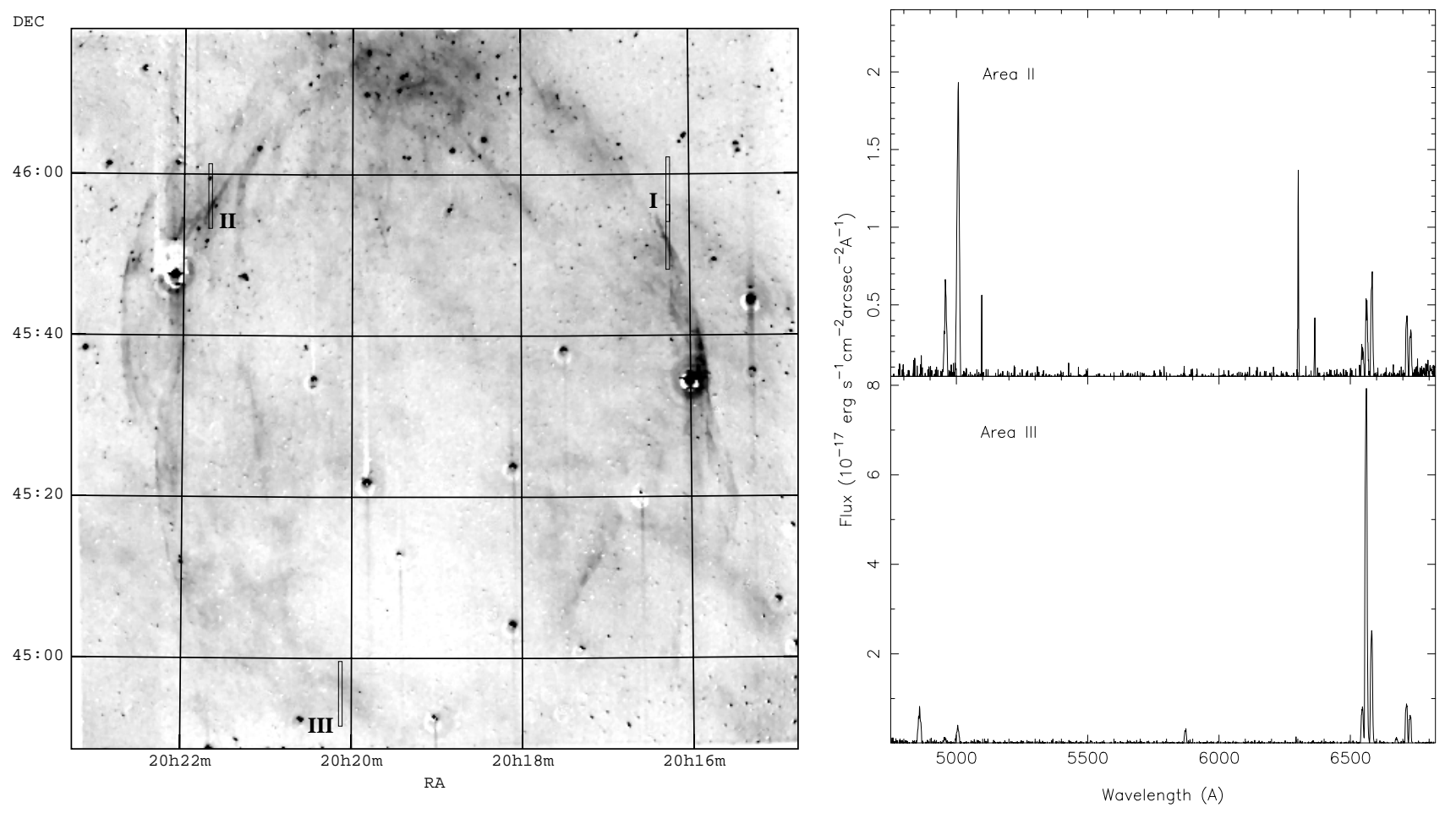

Fig. 3. The medium ionization line of [O III] $5007 \AA$ provides the sharpest view of G $82.2+5.3$ (left). The image has been smoothed to suppress the residuals from the imperfect continuum subtraction and the shadings run linearly from 0 to $30 \times 10^{-17} \mathrm{erg} \mathrm{s}^{-1} \mathrm{~cm}^{-2} \operatorname{arcsec}^{-2}$. The long rectangles indicate the projected positions of the slits on the sky and the numbers designate the areas discussed in the text. The right figure shows single long-slit spectra from areas II and III.
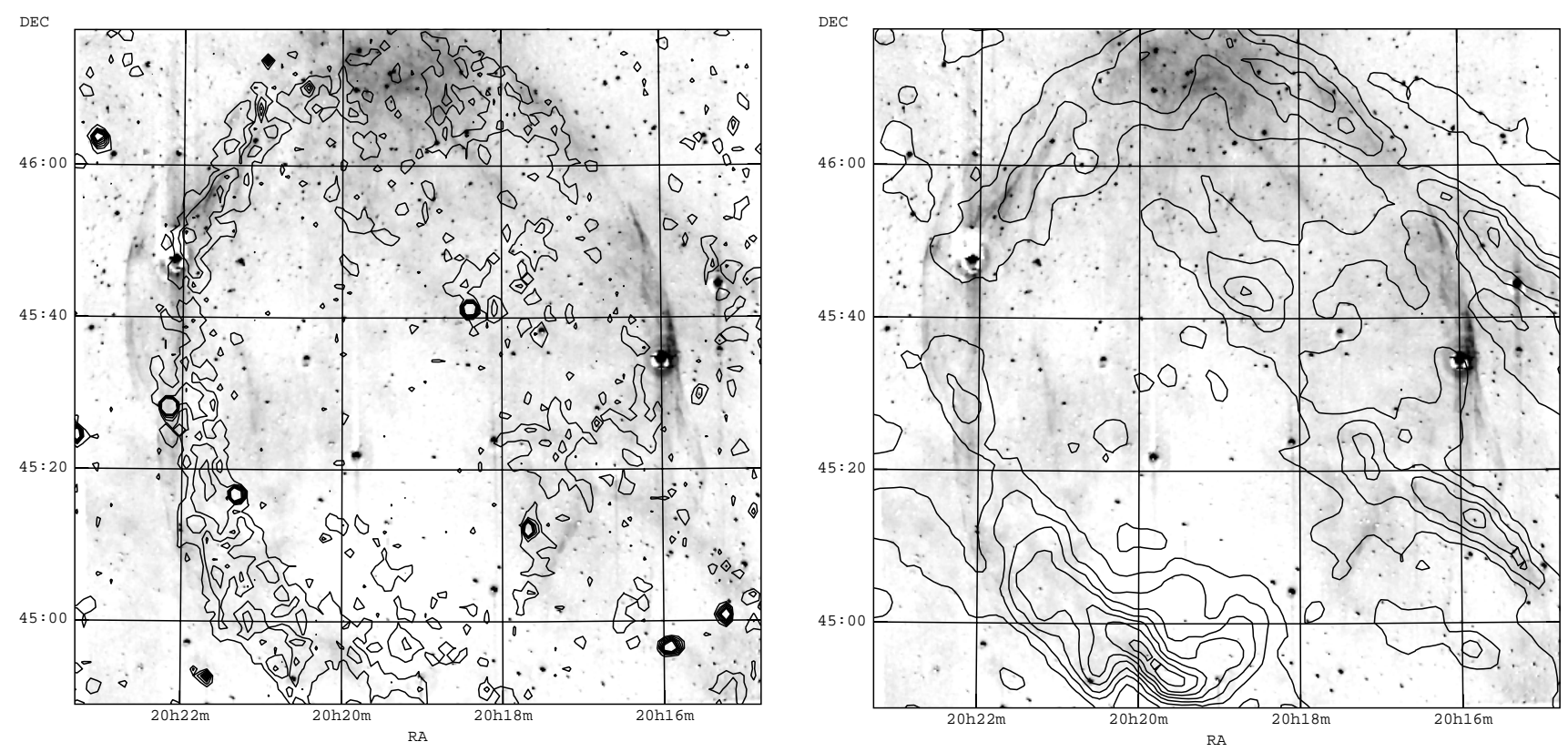

Fig. 4. The correlation between the [O III] emission and the radio emission at $326 \mathrm{MHz}$ (left) and $4850 \mathrm{MHz}$ (right) is shown in this figure. The $326 \mathrm{MHz}$ radio contours (Rengelink et al. 1997) scale linearly from $0.01 \mathrm{Jy} /$ beam to $0.09 \mathrm{Jy} / \mathrm{beam}$, while the $4850 \mathrm{MHz}$ contours scale from $5 \times 10^{-4} \mathrm{Jy} /$ beam to $0.2 \mathrm{Jy} /$ beam (Condon et al. 1994). The optical and radio data are rather well correlated in the east areas of the remnant. The radio emission in the west seems to be weeker and mainly related to the H II region.

from area III may be contaminated by emission from a nearby $\mathrm{H}$ II region, thus causing the low [S II]/ $\mathrm{H} \alpha$ ratio of 0.18 .

Due to the presence of strong H II emission around area I, we report the procedure followed with the spectral analysis. The first set of spectra were taken with the slit crossing the [O III] filament. However, the area along the slit and in the north of the [O III] filament contained very strong H II emission. In order to take into account the diffuse emission present in the field, the second set of spectra were taken with the slit shifted to the north by $6^{\prime}$. In this way the area around $\delta \simeq 46^{\circ} 00^{\prime}$, 
Table 3. Relative line fluxes.

\begin{tabular}{llll}
\hline \hline & I (west) & II (east) & III (south) \\
\hline Line $(\AA)$ & $F^{1,2}$ & $F^{1,2}$ & $F^{1,2}$ \\
$4861 \mathrm{H} \beta$ & $25(19)$ & $15(10)$ & $11.4(66)$ \\
$4959[\mathrm{O} \mathrm{III}]_{1}$ & $34(20)$ & $159(104)$ & $1.5(11)$ \\
$5007[\mathrm{O} \mathrm{III}]_{2}$ & $105(57)$ & $511(293)$ & $5.0(36)$ \\
$5872 \mathrm{He} \mathrm{I}$ & - & - & $3.5(37)$ \\
$6300[\mathrm{O} \mathrm{I}]_{1}$ & - & $75^{3}(32)$ & - \\
$6364[\mathrm{O} \mathrm{I}]_{2}$ & - & $22^{3}(13)$ & - \\
$6548[\mathrm{~N} \mathrm{II}]_{1}$ & $15(18)$ & $44(44)$ & $9.7(95)$ \\
$6563 \mathrm{H} \alpha$ & $100(65)$ & $100(73)$ & $100(520)$ \\
$6584[\mathrm{~N} \mathrm{II}]_{2}$ & $63(55)$ & $141(115)$ & $32.2(260)$ \\
$6678 \mathrm{He} \mathrm{I}$ & - & - & $1.3(17)$ \\
$6716[\mathrm{~S} \mathrm{II}]_{1}$ & $35(39)$ & $78(73)$ & $10.8(113)$ \\
$6731[\mathrm{~S} \mathrm{II}]_{2}$ & $25(29)$ & $56(53)$ & $7.6(82)$ \\
\hline $\mathrm{Absolute} \mathrm{H} \alpha$ flux $^{4}$ & 15.0 & 4.3 & 72 \\
$\mathrm{H} \alpha / \mathrm{H} \beta$ & $4.0(18)$ & $6.7(10)$ & $8.8(65)$ \\
{$[\mathrm{O} \mathrm{III}] / \mathrm{H} \beta$} & $5.6(18)$ & $45(10)$ & $0.57(30)$ \\
{$[\mathrm{S} \mathrm{II}] / \mathrm{H} \alpha$} & $0.6(39)$ & $1.34(56)$ & $0.18(44)$ \\
$\mathrm{I}(6716) / \mathrm{I}(6731)$ & $1.40(23)$ & $1.39(43)$ & $1.42(66)$ \\
\hline$c^{5}$ & $0.38[0.07]$ & $1.1[0.1]$ & $1.41[0.01]$ \\
$E(B-V)$ & $0.25[0.05]$ & $0.73[0.07]$ & $0.94[0.01]$ \\
$n_{\mathrm{e}}$ & $<80 \mathrm{~cm}^{-3}$ & $<30 \mathrm{~cm}^{-3}$ & $<30 \mathrm{~cm}^{-3}$ \\
$V_{\mathrm{s}}$ & $<100 \mathrm{~km} \mathrm{~s}^{-1}$ & $>100 \mathrm{~km} \mathrm{~s}^{-1}$ & - \\
\hline
\end{tabular}

${ }^{1}$ Uncorrected for interstellar extinction.

${ }^{2}$ Listed fluxes are a signal to noise weighted average of the individual fluxes.

${ }^{3}$ Present in only one of the spectra.

${ }^{4}$ In units of $10^{-17} \mathrm{erg} \mathrm{s}^{-1} \mathrm{~cm}^{-2} \operatorname{arcsec}^{-2}$.

5 The logarithmic extinction is determined as $c=1 / 0.331 \times$ $\log \left((\mathrm{H} \alpha / \mathrm{H} \beta)_{\mathrm{obs}} / 3\right)$. Numbers in parentheses represent the signal to noise ratio of the quoted fluxes. The $1 \sigma$ error is given in square brackets for the logarithmic extinction. All fluxes normalized to $F(\mathrm{H} \alpha)=100$.

containing relatively weak $\mathrm{H}$ II emission, was within the slit and was used as background. It is clear that the spectral results from area I should be used under the assumption of constant background intensity. The low $[\mathrm{O} \mathrm{III}] / \mathrm{H} \beta$ ratio of $\sim 6$ implies a shock speed $\sim 100 \mathrm{~km} \mathrm{~s}^{-1}$ or less, while the sulfur line ratio suggests a pre-shock cloud density of a few atoms per $\mathrm{cm}^{3}$ (e.g. Raymond et al. 1988, Osterbrock 1989). The spectrum from area II suggests electron densities below $\sim 80 \mathrm{~cm}^{-3}$, while the strong sulfur line emission may suggest a largely neutral preshock medium. In addition, this spectrum displays very strong [O III] emission relative to $\mathrm{H} \beta$, a characteristic of shocks with incomplete recombination zones (Raymond et al. 1988). This high $[\mathrm{O} \mathrm{III}] / \mathrm{H} \beta$ ratio of $\sim 45$ further suggests shock velocities greater than $\sim 100 \mathrm{~km} \mathrm{~s}^{-1}$.

The $\mathrm{H} \alpha / \mathrm{H} \beta$ ratio of $4.0( \pm 0.2)$ from area I results in a logarithmic extinction of $0.38( \pm 0.07)$ or an $E(B-V)$ of 0.25 $( \pm 0.05)$, while in area II the logarithmic extinction of $1.1( \pm 0.1)$ is equivalent to an $E(B-V)$ of $0.73( \pm 0.07)$. The logarithmic extinction is calculated through the relation $c=1 / 0.331 \times$ $\log \left((\mathrm{H} \alpha / \mathrm{H} \beta)_{\mathrm{obs}} / 3\right)$ and uses the interstellar extinction curve of Kaler (1976) as implemented in the nebular package (Shaw \& Dufour 1995) within the IRAF software. The $E(B-V)$ is calculated with the aid of the relation $E(B-V)=0.664 \times c$ (Kaler 1976; Aller 1984). We note that the signal to noise ratios quoted in Table 3 do not include calibration errors which are $\sim 7 \%$.

\section{X-ray emission from $\mathrm{G} \mathbf{8 2 . 2 + 5 . 3}$}

The field of G 82.2+5.3 was observed by ROSAT in pointed mode on October 26, 1993 (ROR 500286p) for $8 \mathrm{ks}$. The image of this observation is shown in Fig. 5. On purpose we show just the count/pixel image and did not correct for exposure, to illustrate where the remnant is highly underexposed due to the artifacts of the PSPC, clearly seen as a dark bar running from the lower left to the upper right of the image and other ribs, as well as a dark ring 40 arcmin wide around the center. Since the image is not exposure corrected the intensity close to the edge is brighter by a factor of about two than the brightness in the image indicates. The brightness appears quite clumpy and is dominated by a relatively bright bar off-set from the geometric centre to the north by about 9 arcmin. The bar measures about 24 arcmin in the north-south direction and about 18 arcmin along the east-west direction. In Fig. 5 the PSPC image is shown along with contours of the radio emission at $326 \mathrm{MHz}$, which best define the elliptical shape of G 82.2+5.3. It is also evident from this figure that the $\mathrm{X}$-ray emission from the remnant does not fill the PSPC field of view, which is supported by the all-sky image on an even wider scale. Thus it is possible to determine the background from the same observation. Checks with the background taken from the adjacent pointings have been made and the source spectrum does not change. G 82.2+5.3 was also observed by the ASCA satellite on June 12, 1997. The ASCA X-ray telescope was pointed to the central area of G $82.2+5.3$ for a total of $82 \mathrm{ks}$. Given the smaller field of view of the GIS and SIS detectors compared to the ROSAT PSPC, this data can only be used for spectral analysis (see Sect. 4.2).

\subsection{Image analysis}

The first and so far only results of an analysis of the ROSAT data of G 82.2+5.3 were reported by Rho \& Petre (1998), but no images were shown. The X-ray image looks like an SNR broken into pieces resulting into a patchy appearance. The central bar is the brightest component of the remnant and has led Rho \& Petre (1998) to put G 82.2+5.3 in the class of mixedmorphology remnants. First, we have searched the remnant for point sources. The ROSAT source detection algorithm reveals the existence of 31 point sources with a likelihood $>10$ or $4.1 \sigma$ across the face of the remnant. Within a circle of $40^{\prime}$ diameter centered on the apparent SNR centre 17 point-like sources are found out of which four sources do not have optical counterparts down to $m_{B}<22$, with separations from known optical sources of $>10^{\prime \prime}$. The source existence significance of $4.1 \sigma$ corresponds to a source count rate of 0.01 counts $\mathrm{s}^{-1}$ which corrected for an absorption column density of $N_{\mathrm{H}}=4.7 \times 10^{21} \mathrm{~cm}^{-2}$ (Sect. 4.2) corresponds to an unabsorbed (0.2-2.4 keV) energy flux of $\sim 2 \times 10^{-13} \mathrm{erg} \mathrm{cm}^{-2} \mathrm{~s}^{-1}$ for a blackbody source spectrum of $k T_{\mathrm{bb}}=100 \mathrm{eV}$. Of course, with a detection significance 


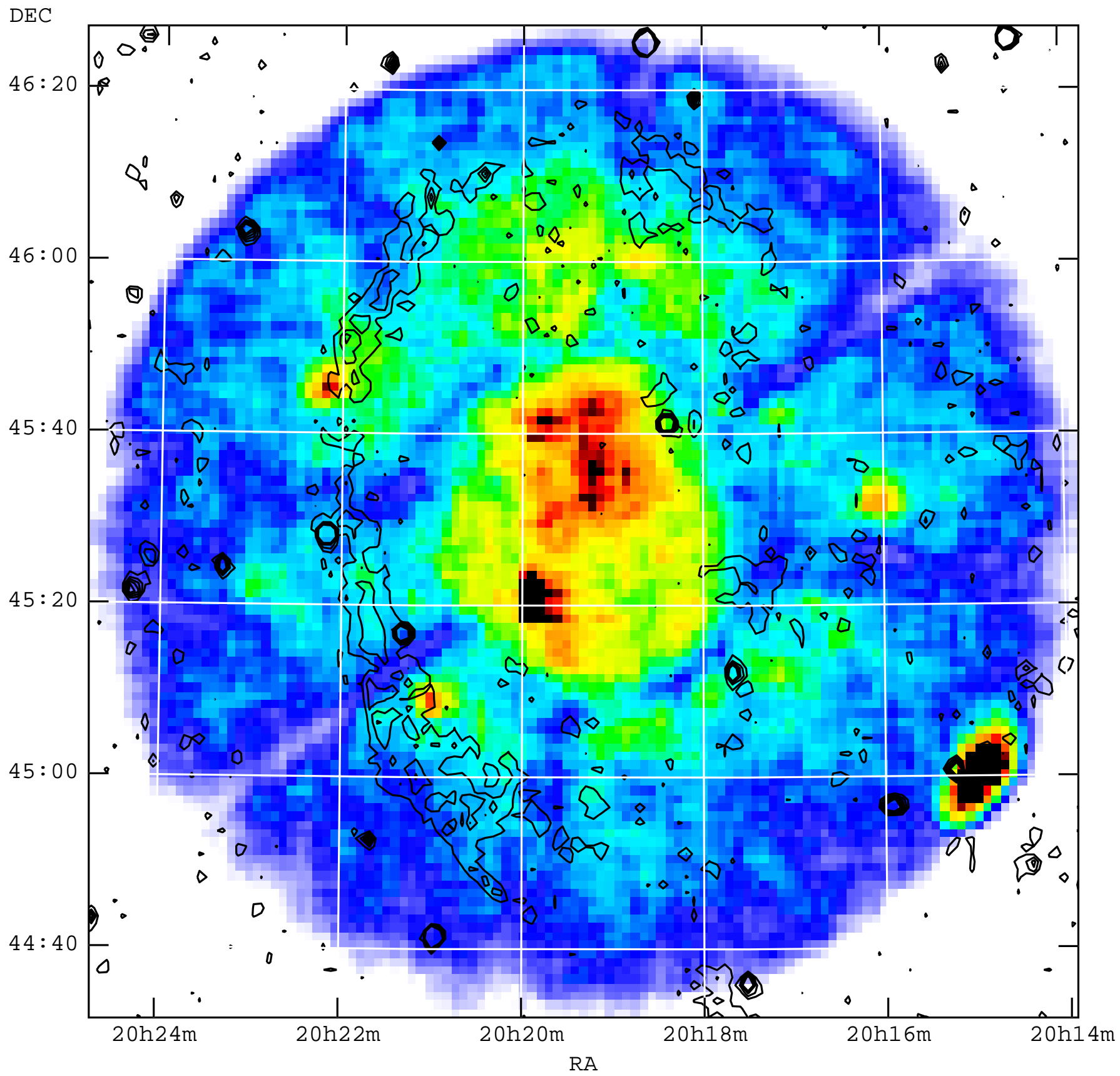

Fig. 5. The raw soft X-ray emission detected in the $8 \mathrm{ks}$ ROSAT pointed observation is shown in this figure. The brightness scales linearly from 2 to 24 counts/pixel and the image has been smoothed with a Gaussian filter with $\sigma=8^{\prime}$. The radio contours at $326 \mathrm{MHz}$ scale also also linearly from 0.015 to $0.09 \mathrm{Jy} /$ beam (Rengelink et al. 1997).

of $4.1 \sigma$ it can not be excluded that these point-like sources are actually statistical fluctuations above the diffuse remnant emission.

Although the surface brightness is quite low, we can integrate over the azimuth and construct its radial profile including every PSPC count independent of energy. Background was subtracted, the profile was corrected for vignetting and the integration was done over $360^{\circ}$ rather than just $40^{\circ}$ which was used by Rho \& Petre (1998). Furthermore we centered the profile on the apparent geometric centre $\left(\alpha \simeq 20^{\mathrm{h}} 19^{\mathrm{m}} 07^{\mathrm{s}}, \delta \simeq 45^{\circ} 31^{\prime} 12^{\prime \prime}\right)$ of the remnant rather than on the brightest patch (cf. 5). It turns out that the density profile can be well described by an exponential law $\left(\propto \mathrm{e}^{-r / r_{0}}\right)$ with a characteristic angular length of $36^{\prime}\left( \pm 3^{\prime}\right)$ up to a distance of $45^{\prime} .5\left( \pm 1^{\prime} .5\right)$. At larger distances the density can be described by the same exponential law but enhanced by a factor of $1.4_{-0.2}^{+0.6}$, which might be interpreted as an indication of limb brightening. The brightness profile is also consistent with a density power law, such that the density scales inversely with the square root of the distance from the center. The actual data allow us to trace the density profile out to an angular distance of $\sim 50^{\prime}$, which we assume to be the radius of the remnant (Fig. 6). In summary, the average matter density 


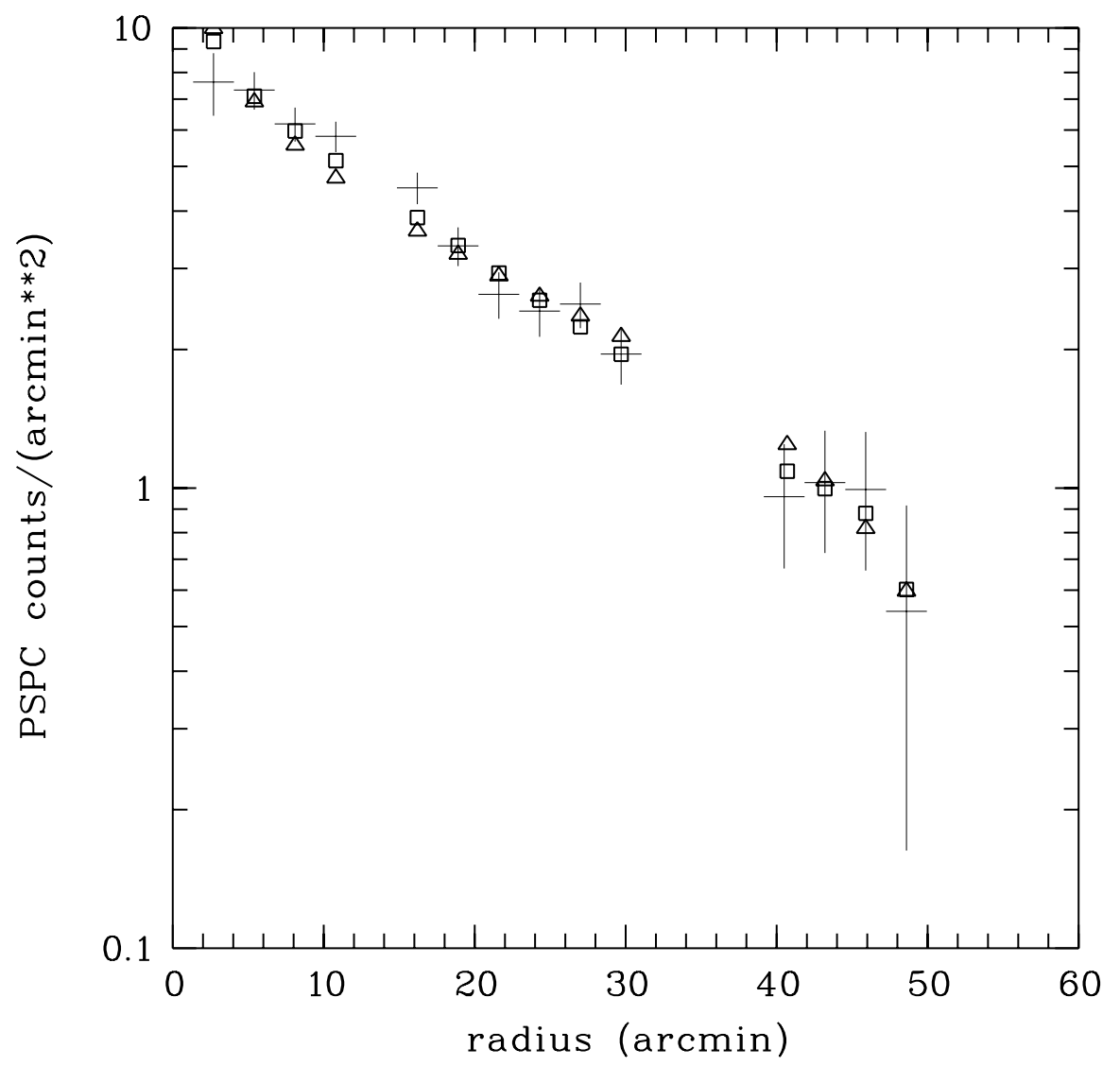

Fig. 6. The brightness profile (crosses) of G 82.2+5.3 derived from the ROSAT data is shown in this figure. The open squares mark the exponential best fit with some limb brightening, while the open triangles mark the power-law best fit. The reader is referred to Sect. 4.1 for more details.

drops by just about a factor of four from the center to the edge. Of course, the radial brightness change could also partially be produced by a radially changing filling factor. The patchiness of the surface brightness distribution is then a matter of local density variations and volume filling factors. This result is quite in contrast to the result of Rho \& Petre (1998), who, however, have chosen a fairly small sector of the remnant (1/9 of the whole perimeter) and probably have centered the profile on the brightest spot.

\subsection{Spectral analysis}

In order to study in as much detail as possible the spectral properties of G 82.2+5.3, we analyzed archival ASCA data and the ROSAT data. Due to the low surface brightness of the object, both the ASCA and ROSAT PSPC data are characterized by low signal to noise, not allowing a spatially resolved spectral analysis. Thus the analysis of the GIS data was restricted to the brightest region within a circle centered on $\alpha \simeq 20^{\mathrm{h}} 19^{\mathrm{m}} 14^{\mathrm{s}}, \delta \simeq$ $45^{\circ} 47^{\prime} 00^{\prime \prime}$ and $7^{\prime} .4$ radius. However, the center of the ASCA SIS detectors is offset by $\sim 6^{\prime}$ with respect to the center of the GIS detectors. Since the SIS data partially overlap the GIS data, counts from a $3^{\prime} \times 3^{\prime}$ square area were extracted from the former detectors.

A non-thermal component is immediately ruled out by the ASCA data since a power-law fit is not acceptable (reduced $\chi^{2}=1.74$ for 197 degrees of freedom). A mekal model fitted simultaneously to the GIS/SIS data with non-cosmic abundances results in a reduced $\chi^{2}$ of 1.15 for 196 degrees of freedom (d.o.f.), which is formally acceptable. However, the presence of a systematic trend in the residuals in the 0.9 to $1.9 \mathrm{keV}$ range suggests its rejection, while including a power-law component does not lead to an improved fit. The GIS/SIS data were also fitted with a vmekal model and a reduced $\chi^{2}$ of 0.99 for 198 d.o.f. (Fig. 7) was obtained. The best fit parameters of this model are given in Table 4. Adding a second component (power-law) does not substantially improve the fit. The vmekal fits also constrain the abundance of $\mathrm{O}(1 \pm 1), \mathrm{Ne}\left(0.1_{-0.1}^{+0.7}\right), \mathrm{Mg}\left(2.8_{-0.7}^{+1.3}\right)$, $\mathrm{Si}\left(4.0_{-1.2}^{+1.2}\right)$ and $\mathrm{Fe}\left(3.5_{-0.8}^{+2.2}\right)$; numbers in parentheses are abundances relative to solar values with $1 \sigma$ errors, which shows that $\mathrm{Mg}, \mathrm{Si}$ and Fe definitely differ from solar values. The $\mathrm{Si}$ line is very clearly seen in both the GIS and SIS spectra (cf. Fig. 7) at around $1.8 \mathrm{keV}$. This is interesting because it might mean that the bright patch, i.e. the near center component, is dominated by ejecta matter rather than circumstellar or interstellar matter. For the ROSAT analysis we used the same field and the same abundances with a one temperature vmekal spectrum. Figure 8 shows the error contours relating the temperature $k T$ and the interstellar absorption column density $N_{\mathrm{H}}$. As the contour lines show there is basically no overlap between the ASCA and the ROSAT fits, except at the $4 \sigma$ level $(99.99 \%$ probability) for each of the two instruments. Clearly a one temperature 

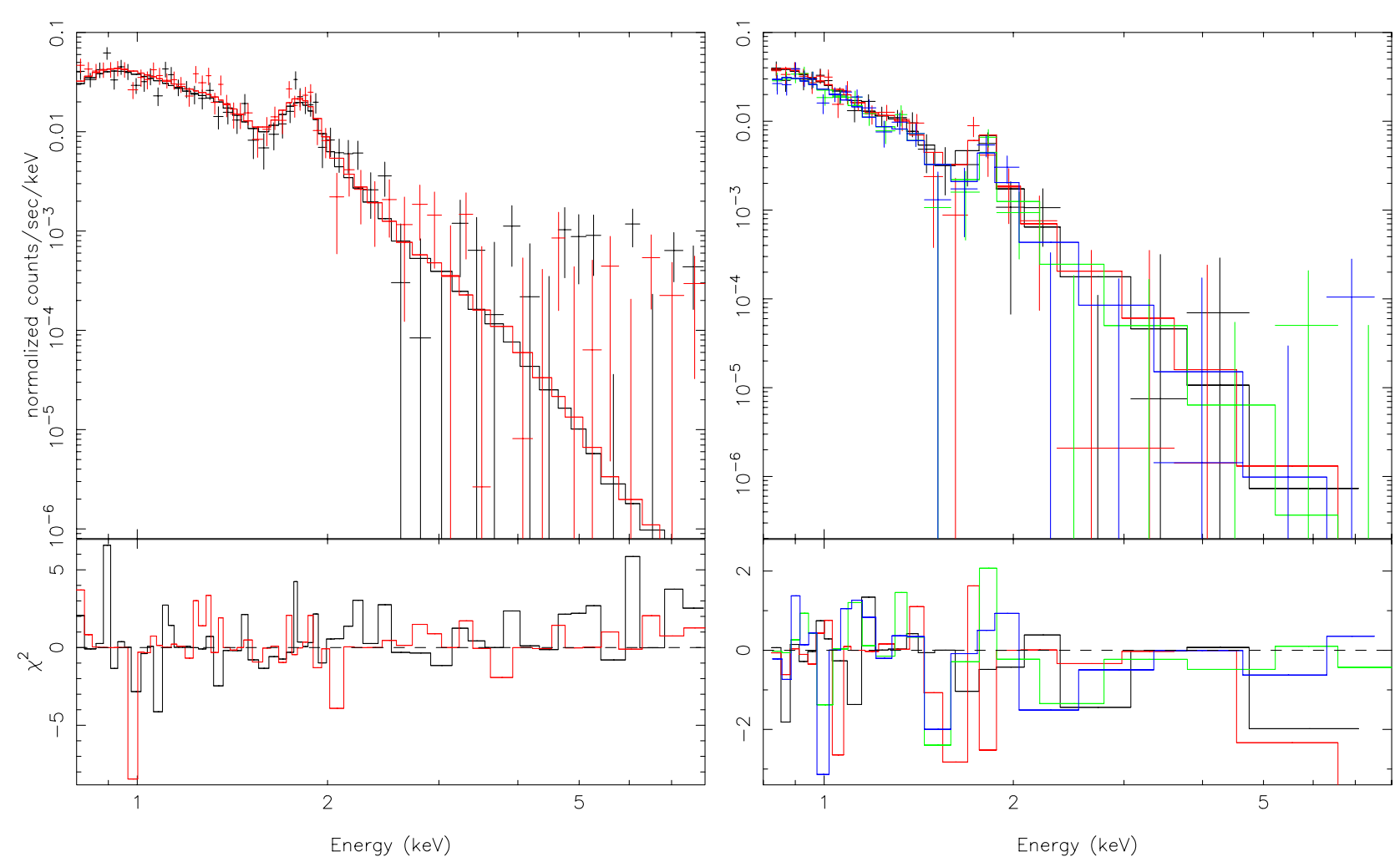

Fig. 7. The ASCA GIS2/3 data are shown in the left figure together with the best fit vmekal model, while the spectra from the SIS detectors are shown in the right figure with the same model (see Sect. 4.2).

Table 4. X-ray spectral fits.

\begin{tabular}{llllll}
\hline \hline Instrument & $N_{\mathrm{H}}$ & $k T$ & $E M$ & $\chi_{\text {red }}^{2}$ & d.o.f. \\
& $10^{21} \mathrm{~cm}^{-2}$ & $\mathrm{keV}$ & $10^{-3} \mathrm{~cm}^{-5}$ & & \\
\hline GIS/SIS & $4.7_{-0.4}^{+0.5}$ & $0.63_{-0.04}^{+0.05}$ & $1.50_{-0.20}^{+0.20}$ & 0.99 & 198 \\
PSPC $^{1}$ & $0.8_{-0.6}^{+6.4}$ & $0.67_{-0.47}^{+0.10}$ & $0.57_{-0.30}^{+0.30}$ & 0.74 & 22 \\
PSPC $^{2}$ & $4.0_{-1.8}^{+4.0}$ & $0.21_{-0.04}^{+0.06}$ & $4.70_{-2.1}^{+4.7}$ & 0.79 & 48 \\
\hline
\end{tabular}

${ }^{1}$ One temperature vmekal model.

2 Two temperature vmekal model; values of low temperature component listed, values of high temperature component restricted to GIS/SIS fit range.

model does not represent the spectrum. Obviously a component with a temperature too low to be observed by ASCA - we have restricted the ASCA analysis to energies $>0.8 \mathrm{keV}-$ has to be added which on the other hand is dominating the ROSAT spectrum, or vice versa. So we fitted the PSPC spectrum with a two component vmekal model, restricting the values of the high temperature, the associated emission measure as well as $N_{\mathrm{H}}$ to the range of $\pm 1 \sigma$ around the best fit found in the ASCA data modeling. The best fit values for the low temperature component are given in Table 4 as well. In summary the high temperature is found to be around $0.6 \mathrm{keV}$ and the low temperature around $0.2 \mathrm{keV}$. The emission measure of the low temperature component is about three times that of the high temperature component. Both the $N_{\mathrm{H}}$ and the value of the low temperature are consistent with the results of Rho \& Petre (1998) but the existence of a high temperature component, already indicated by a deeper analysis of the PSPC spectrum, is very clearly demonstrated by adding the ASCA data to the analysis.

The emission measures of both components allow us to estimate the corresponding densities of the X-ray emitting gas. It is found that, for a distance of $1.6 \mathrm{kpc}$, the average hydrogen number density of the harder component is $\sim 0.03 \mathrm{~cm}^{-3}$, while that of the softer component is $\sim 0.05 \mathrm{~cm}^{-3}$ and both scale inversely with the square root of the distance to the remnant. These numbers adopt an emission volume of $\pi \times(7.4 \text { arcmin })^{2} \times 100$ arcmin, so that the hydrogen number density in $\mathrm{cm}^{-3}$ is $n_{\mathrm{H}}=0.657 \times \sqrt{\left(E M / d_{1.6}\right)}$, with $E M$ the emission measure (cf. Table 4$)$. We note here that a change by a factor of 4 in distance would alter the densities by only a factor of 2. It is clear that the X-ray gas is quite thin and that the remnant expands in an even thinner medium, if we consider the matter inside as being compressed by shocks.

As mentioned above another pointing on $\mathrm{G} 82.2+5.3$ with spectrally useful data has been performed. The spectral analysis of the PSPC pointing centered on the south-east of the remnant shows that the spectrum is fully consistent with that of the central part, and no significant differences can be established. In fact the fit with a one temperature vmekal model produces the same parameter values within $1 \sigma$ error bars. 


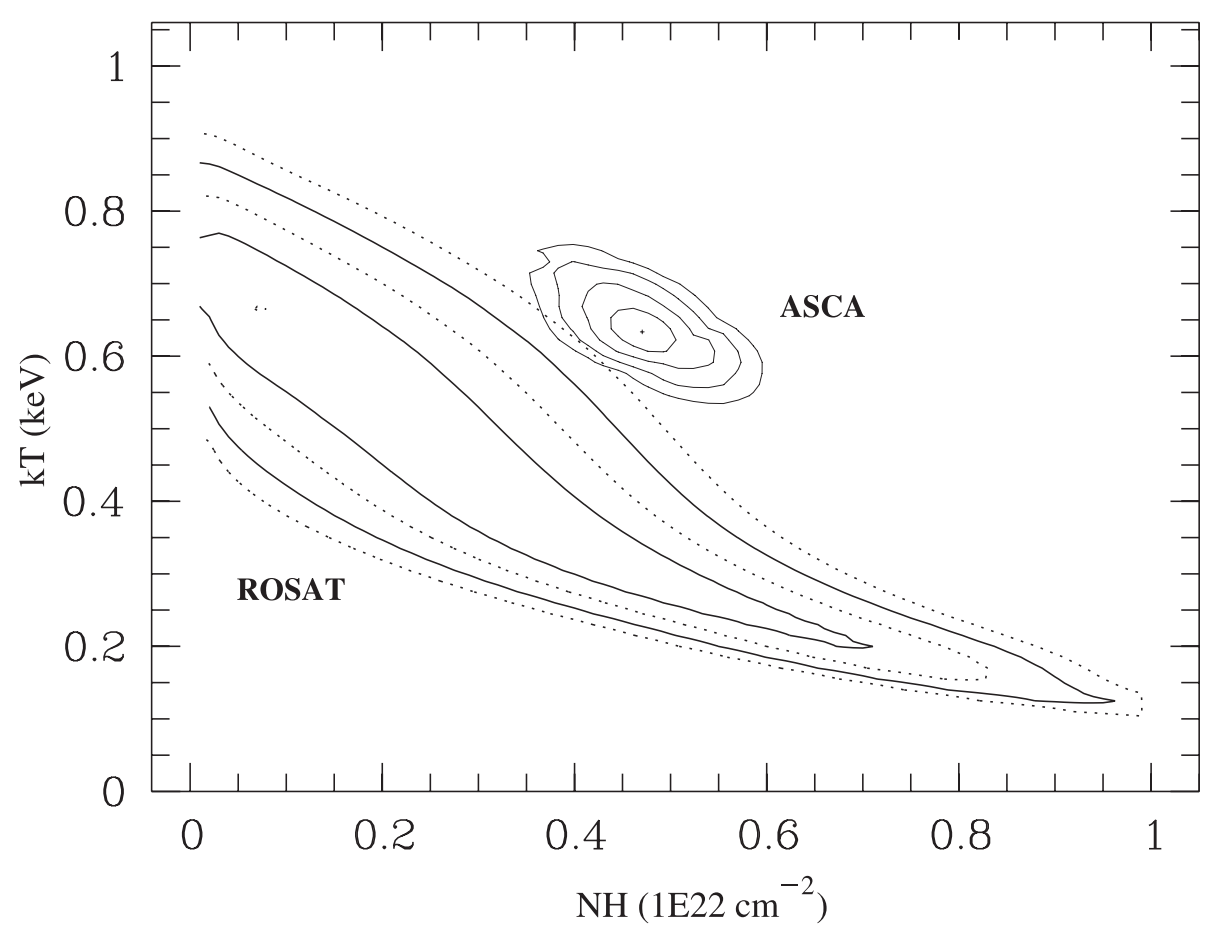

Fig. 8. Best fit and error contours ( $1 \sigma$ to $4 \sigma$ in steps of $1 \sigma)$ for the temperature $(k T)$ and the interstellar absorption column density $N_{\mathrm{H}}$ for one-temperature vmekal models to the ROSAT PSPC spectrum (lower left) and the ASCA GIS/SIS spectra (upper right). Note that there is essentially no overlap, indicating the need for more advanced models.

The existence of a high temperature component cannot unambiguously be demonstrated, it might be present or might be missing. ASCA data on the field are not available.

\section{Discussion}

The supernova remnant $\mathrm{G} 82.2+5.3$ is found in a complex area in the Cygnus constellation where strong $\mathrm{H}$ II emission dominates the red part of the optical spectra. Most of the radio emission is detected in a shell which basically defines the boundaries of the remnant. However, a deficiency of radio emission at the west part of the remnant is present, at least in certain radio frequencies as well as in the X-rays. In this work we present the first flux calibrated CCD images of $\mathrm{G} 82.2+5.3$ in the emission lines of $\mathrm{H} \alpha+[\mathrm{N}$ II $]$, [S II], [O II], and [O III]. In addition, results from a detailed imaging and spectral analysis of the available ROSAT and ASCA pointed observations are presented.

The low ionization images are dominated by the bright $\mathrm{H}$ II region in the field, not allowing the identification of optical emission as emission from the remnant based only on morphological arguments. It is the ratio of the [S II] to $\mathrm{H} \alpha+[\mathrm{N} \mathrm{II}]$ images which provides some evidence on the nature of the emission in this field. We have also detected two filamentary structures, unknown up to now, in the medium ionization line of [O III] in the west and the east parts of the field observed. The two filaments are found at almost opposite sides and display an appreciable degree of curvature. The eastern filament is very well correlated with the radio emission at 326 and $4850 \mathrm{MHz}$ (Fig. 4), while weak radio emission at the location of the western filament is only seen in the $11 \mathrm{~cm}$ maps (Velusamy \& Kundu 1974; Wendker 1971).
The strong differences between the low and medium ionization lines suggest that significant inhomogeneities and density variations in the pre-shock medium are present. The $\mathrm{H} \alpha$, [N II] and [S II] lines being produced in cooler areas behind the shock front are more sensitive to inhomogeneities and density variations than the [O III] line which is produced closer to the shock front (e.g. Hester 1987). The presence of [O III] emission at only certain areas of a remnant seems to be a common characteristic of several remnants like CTB 1 (Fesen et al. 1997) and G 17.4-2.3 (Boumis et al. 2002).

The optical spectrum obtained from area I is a typical spectrum from a complete shock structure suggesting a column density behind the shock front of $\sim 10^{18.5} \mathrm{~cm}^{-2}$ (e.g. Cox \& Raymond 1985; Raymond et al. 1988). However, the large $[\mathrm{O} \mathrm{III}] / \mathrm{H} \beta$ ratio in area II can only be explained by an incomplete shock structure where the shock is traveling with a speed greater than $100 \mathrm{~km} \mathrm{~s}^{-1}$ in an interstellar cloud of medium density like in area I. The swept-up column density is estimated around $10^{18} \mathrm{~cm}^{-2}$ (Raymond et al. 1988). The large sulfur line ratios measured in the long-slit spectra are indicative of low electron densities which in turn imply low pre-shock cloud densities (Fesen \& Kirshner 1980). This is in agreement with the cloud density estimates based on the relative line intensities of the optical spectra and the modeling of Raymond et al. (1988). Currently, the long-slit spectra from area III suggest emission from a photoionized nebula. However, the complex environment of $\mathrm{G} 82.2+5.3$ does not permit an unambiguous determination of the nature of the optical emission in this area and radio observations at different frequencies are required to resolve this issue. 
In an effort to estimate the column density towards G 82.2+5.3, based on the optical data, we use the statistical relation of

$N_{\mathrm{H}}=5.4( \pm 0.1) \times 10^{21} E(B-V) \mathrm{cm}^{-2}$,

given by Predehl \& Schmitt (1995). The estimated neutral hydrogen column density given the observed values of $E(B-V)$ is then in the range of $1-4 \times 10^{21} \mathrm{~cm}^{-2}$, although larger column densities cannot be excluded given the uncertainties in the optical extinction. Furthermore the locations which are accessible for $N_{\mathrm{H}}$ and $E(B-V)$ are different. We formally know $N_{\mathrm{H}}$ only for the central part of the remnant. Nevertheless, the agreement with the absorbing column density derived from the $\mathrm{X}$-ray spectrum $\left(4.7 \times 10^{21} \mathrm{~cm}^{-2}\right)$ is not too bad. The full range of X-ray absorption of $2.2-5.0 \times 10^{21} \mathrm{~cm}^{-2}$ corresponds to $0.4<E(B-V)<0.9$. The $E(B-V)$ color excess in the south is 0.94 and would formally place area III at a larger distance than G 82.2+5.3. However, the low counting statistics from area II and the complex environment do not allow to draw secure conclusions on the relative distance between the remnant and the emission in the south (area III). Additionally, the upper limit on the X-ray absorption does not exclude such a large value of $E(B-V)$ and a correspondingly large distance.

The X-ray emission consists of a patchy disk slightly elongated in the south-north direction, dominated by a bar-like emission close but clearly off-set from the geometric center. The remnant is not obviously limb brightened but a weak shell of emission might be present in the eastern hemisphere. Given the available imaging and spectral $\mathrm{X}$-ray data, we proceed to derive some fundamental parameters of G 82.2+5.3 starting with the Sedov relation. In this framework the remnant is considered to be in the adiabatic phase and estimates for the distance $d$, linear diameter $D$, age $\tau$, luminosity $L_{\mathrm{x}}$, shock velocity $v_{\mathrm{s}}$, pre-shock matter number density $n_{0}$ and the amount of swept-up matter $M_{\mathrm{su}}$ can be derived from the angular diameter, the emission measures and the spectral temperatures (e.g. Pfeffermann et al. 1991). It is interesting to note that a reasonable solution of the Sedov relation cannot be obtained adopting the temperature of the soft component $(0.2 \mathrm{keV}$; see Sect. 4.2$)$ as the temperature at the primary shock front. However, the temperature of the hard component $(\sim 0.6 \mathrm{keV})$, equivalent to a shock velocity of $\sim 720 \mathrm{~km} \mathrm{~s}^{-1}$, does provide a viable solution of the Sedov relation. Assuming a distance of $1.6 \mathrm{kpc}$, the explosion energy would be $0.17 \times 10^{51} \mathrm{erg}$, the age would be $13500 \mathrm{yr}$, the ISM density would be $0.012 \mathrm{~cm}^{-3}$, and the swept up mass would be $24 M_{\odot}$. If, on the other hand, we adopt the nominal value of the explosion energy $\left(1 \times 10^{51} \mathrm{erg}\right)$, then the age would become $26700 \mathrm{yr}$, the current radius would be $50.4 \mathrm{pc}$, the ambient density would be $0.009 \mathrm{~cm}^{-3}$, the swept up mass would be $144 M_{\odot}$, and the remnant should be $\sim 3.3 \mathrm{kpc}$ away from us.

The assumption of pressure equilibrium between the interstellar clouds and the hot gas can provide an independent estimate of the pre-shock cloud density. For a shock velocity of $\sim 100 \mathrm{~km} \mathrm{~s}^{-1}$ into the clouds and an ambient density of $0.012 \mathrm{~cm}^{-3}$, a pre-shock cloud density around $1 \mathrm{~cm}^{-3}$ is estimated, which is compatible with the estimates based on the long-slit optical spectra (see Sect. 3.3).
With the above data, we can discuss the evolutionary status of the remnant in some more detail. Using the calculations of Cox et al. (1999) and Cioffi et al. (1988), it is found that the cooling times exceed $1.3 \times 10^{5} \mathrm{yr}$, i.e. a factor of 10 longer than the age estimated above. Furthermore, with a radius of 50 arcmin at a distance of $1.6 \mathrm{kpc}$ the mean expansion velocity of the remnant over that very long age is just $185 \mathrm{~km} \mathrm{~s}^{-1}$, and with such a low expansion velocity one might wonder about the X-ray emission which has a temperature of at least $0.2 \mathrm{keV}$. Alternatively, the remnant might be at a greater distance, say by a factor of two to three, which might fit the X-ray temperature but it is at the edge of the distance estimates via $N_{\mathrm{H}}$ and $E(B-V)$. Furthermore the measurement of overabundant $\mathrm{Si}$, which indicates that ejecta matter at fairly high temperatures still exists, does probably not favour a very old remnant. Of course it is not excluded that G $82.2+5.3$ has reached the radiative phase, despite the very low ambient density, which we stress is a direct result of the X-ray measurements and is independent of any assumption about the actual evolutionary status of the remnant. In addition, we have examined HI data from the Canadian Galactic Plane survey (CGPS - Taylor et al. 2003). Unfortunately, due to its high galactic latitude of $\sim 5^{\circ}$ only emission from the eastern areas of the remnant was recorded in this survey. So, at least for this part of the remnant, we were not able to identify any features that would point to an HI shell.

The low ambient density may result from the interaction of the stellar wind of the progenitor star with its environment since a typical wind blown bubble is characterized by a radius of $\sim 30 \mathrm{pc}$, a density of $\sim 0.01 \mathrm{~cm}^{-3}$ and a hot interior (e.g. Castor et al. 1975; Weaver et al. 1977). The low surface Xray brightness of the annulus around $\mathrm{G} 82.2+5.3$, if associated with it, would support the suggestion of the wind blown bubble in which the progenitor star of G 82.2+5.3 exploded and its remnant is expanding.

The ASCA spectra of G $82.2+5.3$ show that the central $\mathrm{X}$-ray emission is of thermal origin, while the ROSAT images show a azimuthally averaged brightness profile which is consistent with a slow exponential decrease of the matter density by a factor of about four from the center to the edge. In principle, the evaporating cloud model proposed by White \& Long (1991) or the model of Maciejewski et al. (1999), where a remnant evolves in a stratified medium, can be of relevance to $\mathrm{W}$ 63. This model could probably explain the patchiness and the cloudy structure. Furthermore, heat conduction may apply in order to smooth the temperature distribution in the interior of the remnant and make it look isothermal (e.g. Chevalier 1999). However, with the currently available data we cannot claim the existence of such mechanisms.

At last, there is an interesting aspect which comes to mind after the analysis of the ROSAT PSPC spectra. There may be the possibility that the X-ray emitting gas we see is just a minor fraction of the total matter inside the remnant. If there were gas at temperatures below about $0.05 \mathrm{keV}$, it would have been missed by the X-ray instruments, even if the emission measure would be higher by two to three orders in magnitude, basically because the intersteller absorption column density is so high, 
and this holds for all the mixed morphology remnants listed $\left(N_{\mathrm{H}}>3.8 \times 10^{21} \mathrm{~cm}^{-2}\right)$ by Rho \& Petre (1998).

\section{Conclusions}

We have obtained deep flux calibrated images of G 82.2+5.3. The low ionization images are dominated by the HII region in the field but the medium ionization line of [O III] provides the first clear picture of the remnant. It displays an elliptical shape with filamentary structures in the east and west areas. The correlation between the optical and radio emission supports their association. Long-slit spectra suggest both complete and incomplete recombination zones, shock velocities around $100 \mathrm{~km} \mathrm{~s}^{-1}$, and low electron densities. The X-ray emission region appears to largely be embedded in the optical and radio emission. The X-ray surface brightness is quite patchy, missing obvious limb brightening and is dominated by a bright bar-like emission region which is off-set from the geometric center by $\sim 9^{\prime}$. The X-ray emission is definitely thermal and requires two temperatures of $0.2 \mathrm{keV}$ and $0.63 \mathrm{keV}$. The bright bar region shows overabundant $\mathrm{Mg}$, $\mathrm{Si}$ and $\mathrm{Fe}$, which might indicate still radiating ejecta matter. The azimuthally averaged radial surface profile is consistent with the matter density changing with distance $r$ from the center $\propto \mathrm{e}^{-r / r_{0}}$ with a characteristic angular length of $36^{\prime}$; but it is also consistent with an $r^{-1 / 2}$ density profile. The matter inside the remnant is quite likely structured like a porous cloudy medium. The average matter density derived from the X-ray surface brightness is $\sim 0.05 \times d_{1.6}^{-0.5}$ and $\sim 0.03 \times d_{1.6}^{-0.5}$ for the two spectral components, respectively. It could be slightly higher with filling factors significantly less than 1 . From the X-ray and optical measurements we have determined some parameters of the SNR, but the evolution of the remnant into the mixed-morphology state is still difficult to understand. If we apply the "Sedov analysis" approach, which would assume the remnant to be in the adiabatic phase, the remnant is at a distance between $1.6 \mathrm{kpc}$ and $3.3 \mathrm{kpc}$ with an age of 13.5 and 26.7 kyrs. These results appear reasonable, particularly because the distance is consistent with that determined by Rosado \& Gonzales (1981). But the remnant shows neither the expected density profile nor a strong limb brightening for a constant ambient density on which the "Sedov analysis" is based. In addition, we determine the temperature only at the center and not at the limb which is what is required for the application of the "Sedov analysis". On the other hand, the assumption that the remnant is in the radiative phase entering the shell-forming phase is in conflict with the very low density of the X-ray emitting plasma and the excessive long radiative cooling time involved. Although the low matter density itself does not completely rule out that the remnant has reached the radiative phase it would imply an even lower density, greater age and much larger distance at the edge of the upper limits obtained from $N_{\mathrm{H}}$ and $E(B-V)$. These conclusions are primarily based on the low density of the X-ray plasma but the optical filaments demonstrate the existence of matter of preshock densities of one or a few atoms $\mathrm{cm}^{-3}$ and shock velocities of $100 \mathrm{~km} \mathrm{~s}^{-1}$. Whether these filaments are located around the perimeter of the remnant or inside of it is not clear. But matter at these densities and velocities of $>100 \mathrm{~km} \mathrm{~s}^{-1}$ should emit soft X-rays or EUV radiation. The fact that we do not see such radiation puts an upper limit on the temperature and the emission measure, or electron density, of this component in order to stay "undetected" in the PSPC observations which depend on $N_{\mathrm{H}}$. Adopting the observed value of $N_{\mathrm{H}}$ the pre-shock electron density could be as high as $1 \mathrm{~cm}^{-3}$ for $\log (T)=5.8$ or $k T=55 \mathrm{eV}$. Interestingly, the radiative cooling time is $25 \mathrm{kyrs}$, and an HI shell might not have formed yet. We have not found any evidence for an HI shell, but this is not conclusive as the full area around the remnant has not been imaged so far. Even higher electron densities up to $20 \mathrm{~cm}^{-3}$ with $\log (T)=5.5$ are possible for the soft X-rays/EUV to escape detection but the radiative cooling time gets as short as $10 \mathrm{kyrs}$, and the probability to have caught a remnant in such a brief transition phase gets increasingly lower. If most of the dense matter were predominantly located around the perimeter of the remnant it might represent the shock heated matter of parts of the inner edge of a stellar wind-blown bubble, which might still be larger than the remnant, indicated by the low-level X-ray emission surrounding the remnant as seen in the ROSAT All-sky survey. If the supernova had a stellar wind-blown bubble, the low electron density derived from the X-ray measurements is easy to understand as it matches the prediction of densities in such bubbles. The temperature and the X-ray surface brightness distribution observed in the remnant are then a consequence of the temperature and matter density distributions in the bubble prior to the supernova shock, both of which change substantially with distance from the progenitor star (Weaver et al. 1977).

Acknowledgements. We thank the referee for careful and productive comments and pointing us to the ASCA data; we are grateful to Emi Miyata for her advice on analysing the ASCA data. Skinakas Observatory is a collaborative project of the University of Crete, the Foundation for Research and Technology-Hellas and the Max-PlanckInstitut für Extraterrestrische Physik. This research has made use of data obtained through the High Energy Astrophysics Science Archive Research Center Online Service, provided by the NASA/Goddard Space Flight Center. The data from the Canadian Galactic Plane Survey were obtained from the Canadian Astronomy Data Centre (where F.M. is a guest user) which is operated by the Herzberg Institute of Astrophysics of the National Research Council Canada.

\section{References}

Aller, L. H. 1984, Physics of thermal gaseous nebulae (D. Reidel Publishing Company)

Angerhofer, P. E., Becker, R. H., \& Kundu, M. R. 1977, A\&A, 55, 11

Boumis, P., Mavromatakis, F., \& Paleologou, E. V. 2002, A\&A, 385, 1042

Castor, J., McCray, R., \& Weaver, R. 1975, ApJ, 200, L107

Chevalier, R. A. 1999, ApJ, 511, 798

Cioffi, D. F., McKee, C. F., \& Bertschinger, E. 1988, ApJ, 334, 252

Condon, J. J., Broderick, J. J., Seielstad, G. A., Douglas, K., \& Gregory, P. C. 1994, AJ, 107, 1829

Cox, D. P., \& Raymond, J. C. 1985, ApJ, 298, 651

Cox, D. P., Shelton, R. L., Maciejewski, W., et al. 1999, ApJ, 524, 179

Fesen, R. A., \& Kirshner, R. P. 1980, ApJ, 242, 1023

Fesen, R. A., Winkler, P. F., Rathore, Y., et al. 1997, AJ, 113, 767

Hartigan, P., Raymond, J., \& Hartmann, L., 1987, ApJ, 316, 323

Hamuy, M., Walker, A. R., Suntzeff, N. B., et al. 1992, PASP, 104, 533 
Hamuy, M., Suntzeff, N. B., Heathcote, S. R., et al. 1994, PASP, 106, 566

Hester, J. 1987, ApJ, 314, 187

Kaler, J. B. 1976, ApJS, 31, 517

Lasker, B. M., Russel, J. N., \& Jenkner, H. 1999, in the HST Guide Star Catalog, version 1.1-ACT, The Association of Universities for Research in Astronomy, Inc.

Lynds, B. T. 1962, ApJS, 7, 1

Lynds, B. T. 1965, ApJS, 12, 163

Mavromatakis, F., Boumis, P., \& Paleologou, E. V. 2002, A\&A, 383, 1011

Maciejewski, W., \& Cox, D. P. 1999, ApJ, 511, 792

Osterbrock, D. E. 1989, Astrophysics of gaseous nebulae (W. H. Freeman \& Company)

Parker, A. R., Gull, T. R., \& Kirshner, R. P. 1979, An emission line survey of the Milky Way, NASA SP-434

Pfeffermann, E., Aschenbach, B., \& Predehl, P. 1991, A\&A, 246, L28
Predehl, P., \& Schmitt, J. H. M. M. 1995, A\&A, 293, 889

Raymond, J. C., Hester, J. J., Cox, D., et al. 1988, ApJ, 324, 869

Rengelink, R. B., Tang, Y., de Bruyn, A. G., et al. 1997, A\&AS, 124, 259

Rho, J., \& Petre, R. 1998, ApJ, 503, L167

Rosado, M., \& Gonzalez, J. 1981, Rev. Mex. Astr. Astrof., 5, 93

Sabbadin, F. 1976, A\&A, 51, 159

Shaw, R. A., \& Dufour, R. J. 1995, PASP, 107, 896

Taylor, A. R., Gibson S. J., Peracaula, M., et al. 2003, AJ, 125, 3145

Uyaniker, B., Fürst, E., Reich, W., Aschenbach, B., \& Wielebinski, R. 2001, A\&A, 371, 675

Velusamy, T., \& Kundu, M. R. 1974, A\&A, 32, 375

White, R. L., \& Long, K. S. 1991, ApJ, 373, 543

Weaver, R., McCray, R., Castor, J., Shapiro, P., \& Moore, R., 1977, ApJ, 218, 377

Wendker, H. J. 1971, A\&A, 13, 65 